\title{
Coronal holes and the solar polar field reversal
}

\begin{abstract}
I. A. Bilenko*
Sternberg State Astronomical Institute, Universitetsky pr. 13, 119899 Moscow, Russia

Received 5 February 2002 / Accepted 5 September 2002

Abstract. The relationship between the solar polar magnetic field reversal and coronal hole evolution is investigated for the time interval from 1996 to 2001. The distribution of coronal holes shows some evolutionary changes in relation to the polarity reversals, and these changes appear to be coordinated with changes in the global magnetic field structure, suggesting that the polar reversal originates from global processes. There are periods when the character of the coronal hole distribution on the solar disk changes significantly, indicating that the magnetic field structure changes. These can be interpreted as periods of rearrangement of the multipole magnetic field structure. It is found that the evolution of the geometric structure of the photospheric magnetic field during these periods is not characterized by a continuous transition from one dominant structure to another, but by relatively sudden rearrangement of the dominant geometrical structure of the magnetic field. These coronal holes and photospheric magnetic field rearrangements coincide with the polar photospheric magnetic field strength variations. The minimum phase of the solar cycle is dominated by the dipole component of the global solar magnetic field. The zonal magnetic field structure and zonal non-polar coronal hole distribution existed at that time. The sectorial magnetic field structure appears when the polar field strength reaches 0.7 of its maximum value in the corresponding hemisphere. This structure was established at different times in each hemisphere. In the north hemisphere it has existed since November 1998 and in the south hemisphere it has existed since October 1997. The rearrangement from one configuration to another occurs during a short time period, about $1 \div 2$ solar rotations. The coronal hole number and area evolution indicates a redistribution of positive-polarity and negative-polarity photospheric magnetic fields inside these longitudinal sectors, which reflects the solar polar field reversal.
\end{abstract}

Key words. Sun: activity - Sun: magnetic fields - Sun: corona - Sun: photosphere

\section{Introduction}

The reversal of the polar magnetic field of the Sun requires that each polar magnetic field must be replaced by one of opposite polarity. The standard model for the changing solar cycle magnetic field (Leighton 1964; DeVore \& Sheeley 1987; Sheeley et al. 1987) supposes that the large-scale fields are by-products of active regions and that they are formed by differential rotation, meridional flows, and supergranule motions. These fields drift polewards and change the sign of the polar magnetic field. This process is described by the surface flux transport model. This model faces some difficulties. The weakness of the polar magnetic fields and their proximity to the solar limb make direct field measurements difficult. An alternative method of studying the polar magnetic field evolution is afforded by analyzing filaments, polar faculaes and coronal holes. Properties of a latitude zonal component of the large-scale solar magnetic field were analyzed on the basis of $\mathrm{H}_{\alpha}$ charts (Makarov et al. 1989). In the article by Makarov \& Tlatov (2000) it was shown that the large-scale magnetic field is not a product of active region disintegration.

The latitudinal distribution and poleward migration of prominences reflects the clear relation to the polar field

\footnotetext{
^ e-mail: belenko@sai.msu.ru
}

reversal (Makarov et al. 1983a, 1983b). Bumba et al. (1995) studied mutual relations between the evolution of coronal holes, active regions and development of the large-scale distribution of solar magnetic fields during 1991-1992.

As coronal holes are mainly found on unipolar magnetic fields with one predominant polarity (Harvey et al. 1982; Timothy et al. 1975; Varsic et al. 1999), they can be used as good tracers for the analysis of the evolution of photospheric magnetic field distribution. Coronal holes first were observed by Waldmeier as regions with depressed emission in the $\lambda=5303 \AA$ line. He also investigated cyclic variations of the polar coronal holes and their association with polar faculaes and filaments (Waldmeier 1981) for four cycles (1940-1978). Kulěár \& Sýkora (1994) analyzed long-term and large-scale regularities in latitudinal and longitudinal distribution of coronal holes, situated within $\pm 60^{\circ}$ heliographic latitude, and that of the green corona low brightness regions for each Carrington rotation for the period of 1970-1991. Coronal holes were divided into equatorial coronal holes and polar coronal holes according to their location on the solar disk (Sanchez-Ibarra \& Barraza-Paredes 1992). It was found that the long-lived equatorial coronal holes have approximately the same differential rotation as sunspots, and long-lived polar coronal holes show a rigid rotation (Ikhsanov \& Ivanov 1999). They drew a conclusion about the existence of two types of large-scale solar 
magnetic fields. Webb et al. (1984) examined the evolution of the polar magnetic field around sunspot maximum and coronal hole redevelopment around the poles for solar cycles 21 and 22 using synoptic charts. They found that the process of polarity reversal and redevelopment of polar holes is discontinuous, occurring in 2 or 3 longitude bands and there is a persistent asymmetry in these processes between the two hemispheres; the polarity reversal in the two hemispheres is offset by 6 months to 1.5 yr. Fox et al. (1998), analyzing the reversal of the solar polar magnetic field in cycles 21 and 22, have suggested that the polar reversal originates from global processes rather than from local magnetic flux annihilation. Coronal holes are associated with open magnetic field regions. The structure and cyclic variation of the photospheric open magnetic field regions were analyzed by Obridko \& Shelting (1999) and by Levine (1982).

The aim of this work is to analyze the changes of the spatial and temporal distributions of coronal holes on the solar disk during 1996-2001, their relation to the solar polar field reversal, and evolution of the solar magnetic field around sunspot maximum, when the polar fields change polarity and coronal holes of a new polarity redevelop around the poles, using the daily data and spherical harmonic analysis. In this study, the polar magnetic field strength variations are studied in relation to coronal holes and associated magnetic field evolutionary changes. The asymmetry of the north pole and the south pole polarity reversal is discussed. An understanding of the physical processes that control the reversal of the polar fields should provide some insight into the nature of the solar activity.

\section{Analysis and results}

Other than magnetogram studies, the evolution of the unipolar photospheric magnetic fields can be studied by analyzing the coronal hole distribution. Coronal holes are used to trace the poleward motion of the unipolar photospheric magnetic fields and the redevelopment of the new-polarity polar holes. The daily full-disk magnetograms and coronal hole maps in the $\lambda=10830 \AA$ line of the Kitt Peak NSO were used.

The boundaries of the $\lambda=10830 \AA$ coronal holes are less distinct than those shown in X-ray images, and the boundary of a particular coronal hole varies significantly every day. The number of non-polar coronal holes increases during the rising phase of the solar cycle (Belenko 2001). Locations of individual coronal holes change significantly on the solar disk every day. Coronal hole coordinates were calculated for each coronal hole once per rotation to analyze these changes. Several coronal holes may be observed during a rotation. The sign of the corresponding photospheric magnetic field was taken into account. Coordinates of east, north, west, and south points for each nonpolar coronal hole and the largest polar coronal hole projections were calculated to evaluate their locations on the solar disk and their area. The center coordinates were then calculated for each coronal hole. The standard procedure was applied to transform these coordinates from the two-dimensional $x-y$ coordinate system of the projected solar disk image to a Carrington heliocentric spherical coordinate system. The constants necessary for this transformation are the size of the observed solar disk and the solar ephemeris data on the day of observation. Figure 1 shows the distribution of non-polar coronal hole center latitudes and the largest polar coronal hole projections from 1996 to 2001. Diamonds represent the centers of non-polar coronal holes associated with the negative-polarity photospheric magnetic fields and asterisks those associated with the positivepolarity photospheric magnetic fields. Vertical lines represent maximum north-south extents of non-polar coronal holes and of the polar coronal hole projections. Figure 1a shows the total distribution of non-polar coronal holes and the polar coronal hole projections. Figure $1 \mathrm{~b}$ shows the distribution of nonpolar coronal holes and the polar coronal hole projections associated with the positive-polarity photospheric magnetic fields. Figure 1c shows the distribution of non-polar coronal holes and the polar coronal hole projections associated with the negativepolarity photospheric magnetic fields. Figure 2 shows the same distributions for each year. Figure 3 shows some combined images of magnetograms and coronal hole maps as an example of the coronal hole redistribution, which reflects the redistribution of photospheric magnetic fields related to the solar polarity reversal. The left column shows the coronal holes associated with the negative-polarity photospheric magnetic fields. The right column shows the coronal holes associated with the positivepolarity photospheric magnetic fields. From Figs. 1 and 2 we find that there are three different periods of the coronal hole distribution on the solar disk during 1996-2001. The first period lasted from the beginning of 1996 to the middle of 1997. Non-polar coronal holes were located at low latitudes during that time interval. Coronal holes associated with the positivepolarity photospheric magnetic fields were, in general, located in the north hemisphere and those associated with the negativepolarity photospheric magnetic fields were, in general, located in the south hemisphere. The calculated center location latitudes of the long-lived and newly created non-polar coronal holes associated with positive-polarity photospheric magnetic fields show a drift toward the south hemisphere during this period (Fig. 1b). New coronal holes were created at southern latitudes. The coronal holes associated with the negative-polarity photospheric magnetic fields were, in general, located in the south hemisphere. New ones were created at higher northern latitudes (Fig. 1c). The drift of center location latitudes reflects the photospheric magnetic field redistribution.

The second period lasted until the end of 1998. Polar coronal holes were observed both in the north and the south. During that time the so-called hemisphere polarity rule (the polarity of the coronal-hole-associated photospheric magnetic fields match the polarity of the polar field in that hemisphere (Bohlin \& Sheeley 1997) held for the majority of coronal holes. This rule was violated increasingly by small coronal holes of opposite polarity (Figs. 1, 2). There are small coronal holes associated with the negative-polarity photospheric magnetic fields in the north hemisphere, and ones associated with the positivepolarity photospheric magnetic fields in the south hemisphere. Projections of the polar coronal holes arranged often in the direction of holes of the same polarity at low latitudes or those located in the other hemisphere. Non-polar coronal holes were, in general, located at the active region latitudes. Such a coronal hole distribution reflects a zonal solar magnetic field structure existing at that time. 

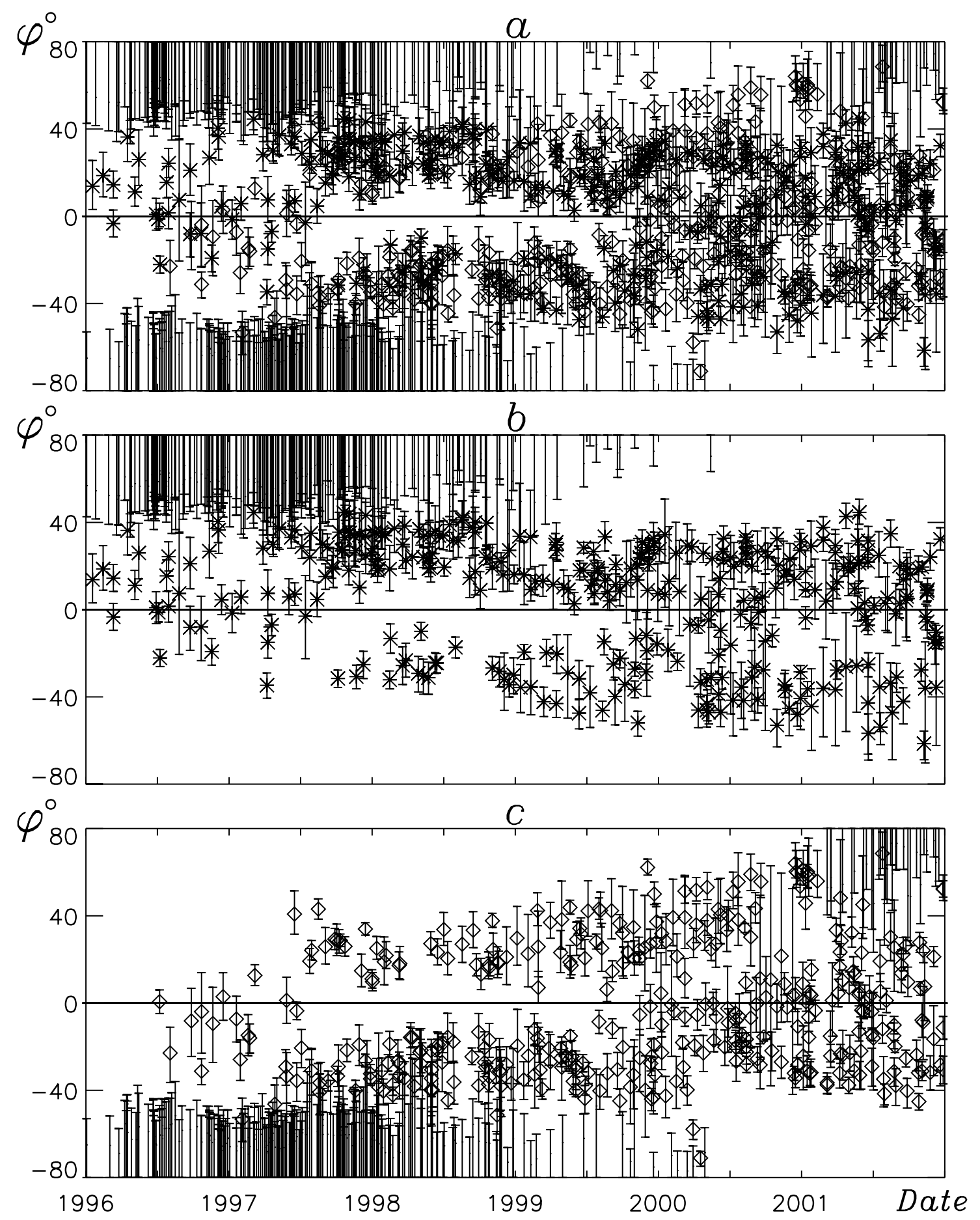

Fig. 1. Distributions of coronal hole center locations and polar coronal hole projections on the solar disk from 1996 to 2001. Diamonds represent the centers of non-polar coronal holes associated with the negative-polarity photospheric magnetic fields and asterisks, those associated with the positive-polarity photospheric magnetic fields. Vertical lines represent the maximum north-south extent of non-polar coronal holes and that of the polar coronal hole projections.

During the third period, the number and area of coronal holes associated with negative-polarity photospheric magnetic fields increased in the north hemisphere and the area and number of coronal holes associated with the negativepolarity photospheric magnetic field located in the south hemisphere decreased. Center latitudes of coronal holes were located at higher latitudes in the north hemisphere nearer to the north pole, the pole of opposite polarity. North pole positive-polarity coronal holes disappeared at the beginning of 2000, about the time of sunspot maximum. Coronal holes associated with the negative-polarity photospheric magnetic fields reached their maximum latitudes at the beginning of 2001 and the new-polarity north polar coronal hole was created at that time. The analogous process took place for 


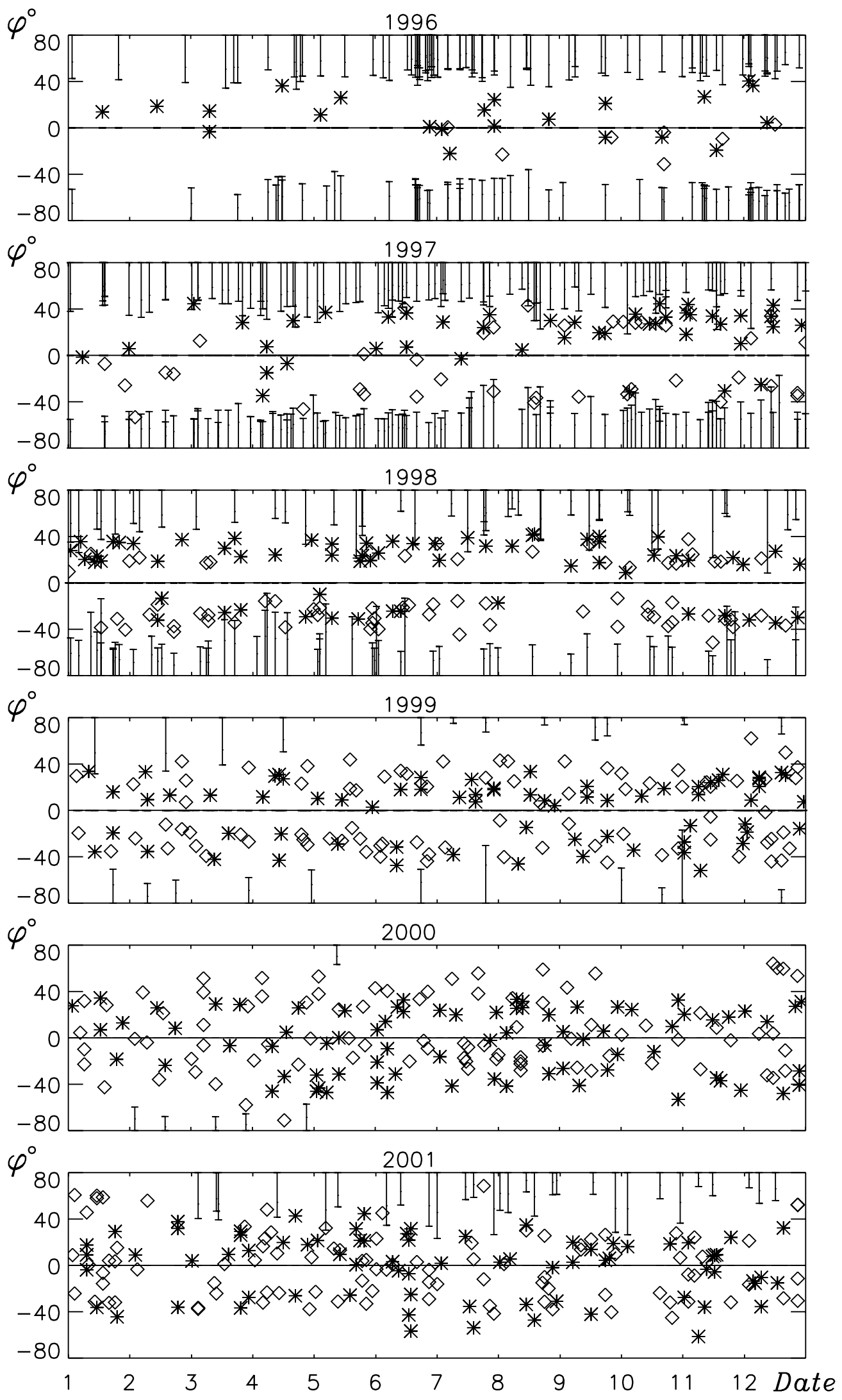

Fig. 2. Same as Fig. 1 but for each year. The years are indicated on the top. See Fig. 1 for explanation of the symbols.

the coronal holes associated with the positive-polarity photospheric magnetic field, but the time of the new positive- polarity, the south pole coronal hole creation was shifted approximately by 1 year (Fig. 3). Since October 1997, 


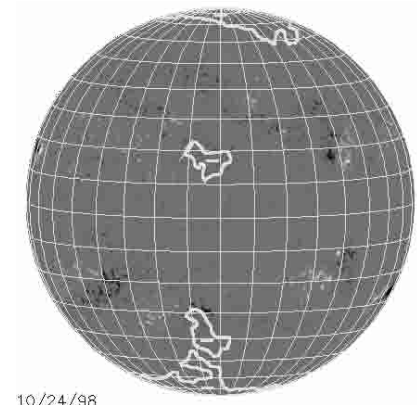

$10 / 24 / 98$

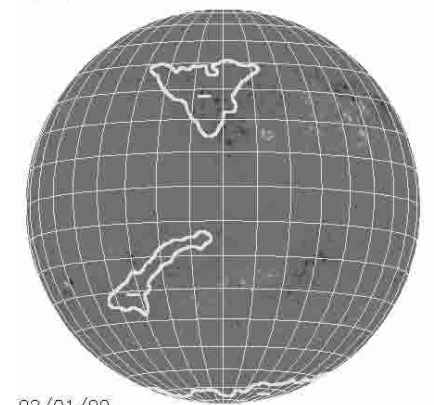

02/01/99

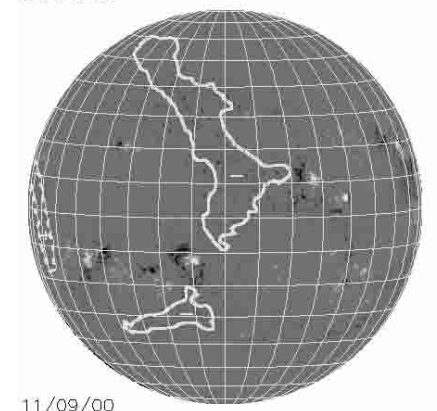

$11 / 09 / 00$
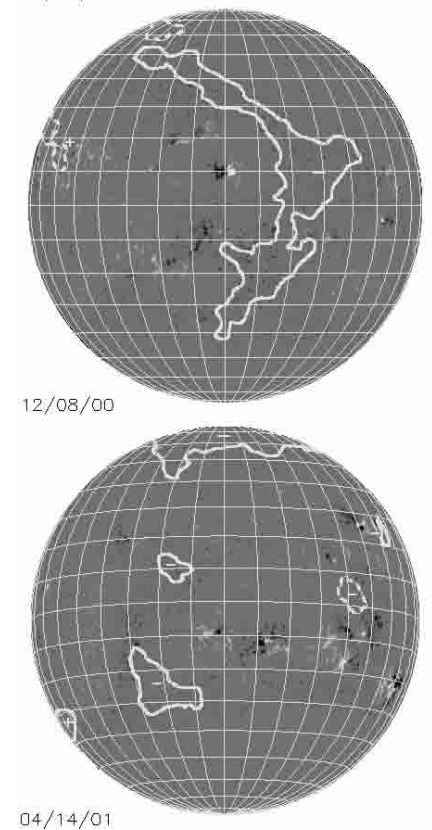
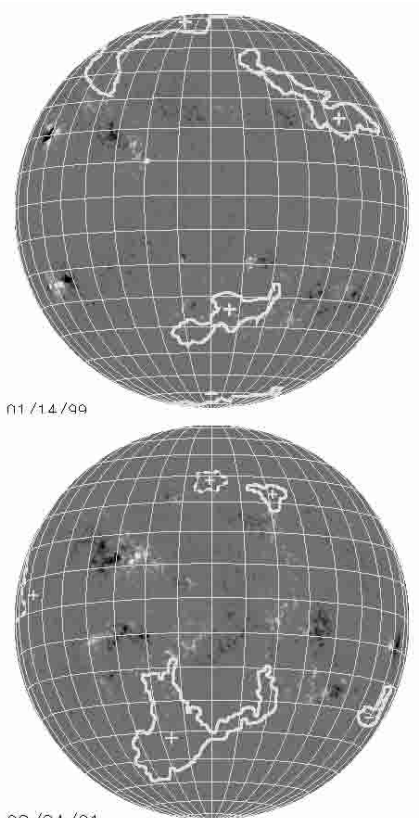

$02 / 24 / 01$

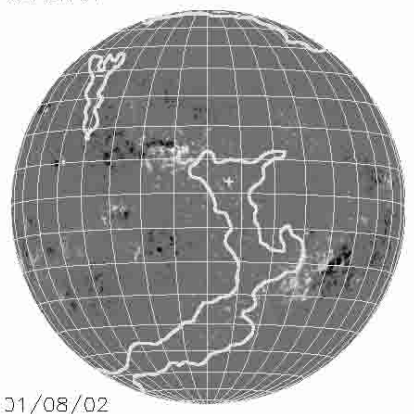

$31 / 08 / 02$

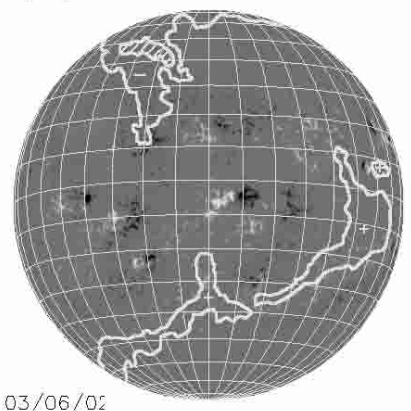

$03 / 06 / 02$

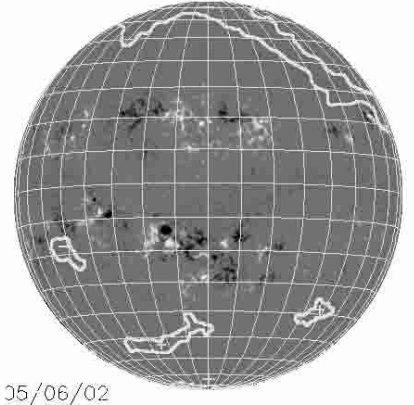

Fig. 3. Examples of the redistribution of coronal holes associated with the negative-polarity photospheric magnetic fields (left column), and that of coronal holes associated with the positive-polarity photospheric magnetic fields (right column) and the polar coronal hole sign changes during 1998-2002.

coronal holes associated with negative-polarity and positivepolarity photospheric magnetic fields began to alternate in approximately 13 day intervals in the south hemisphere
(Fig. 2, 1997). In the north hemisphere such an alternating mode has been seen since November 1998 (Fig. 2, 1998). A regular alternation of unipolar coronal hole 

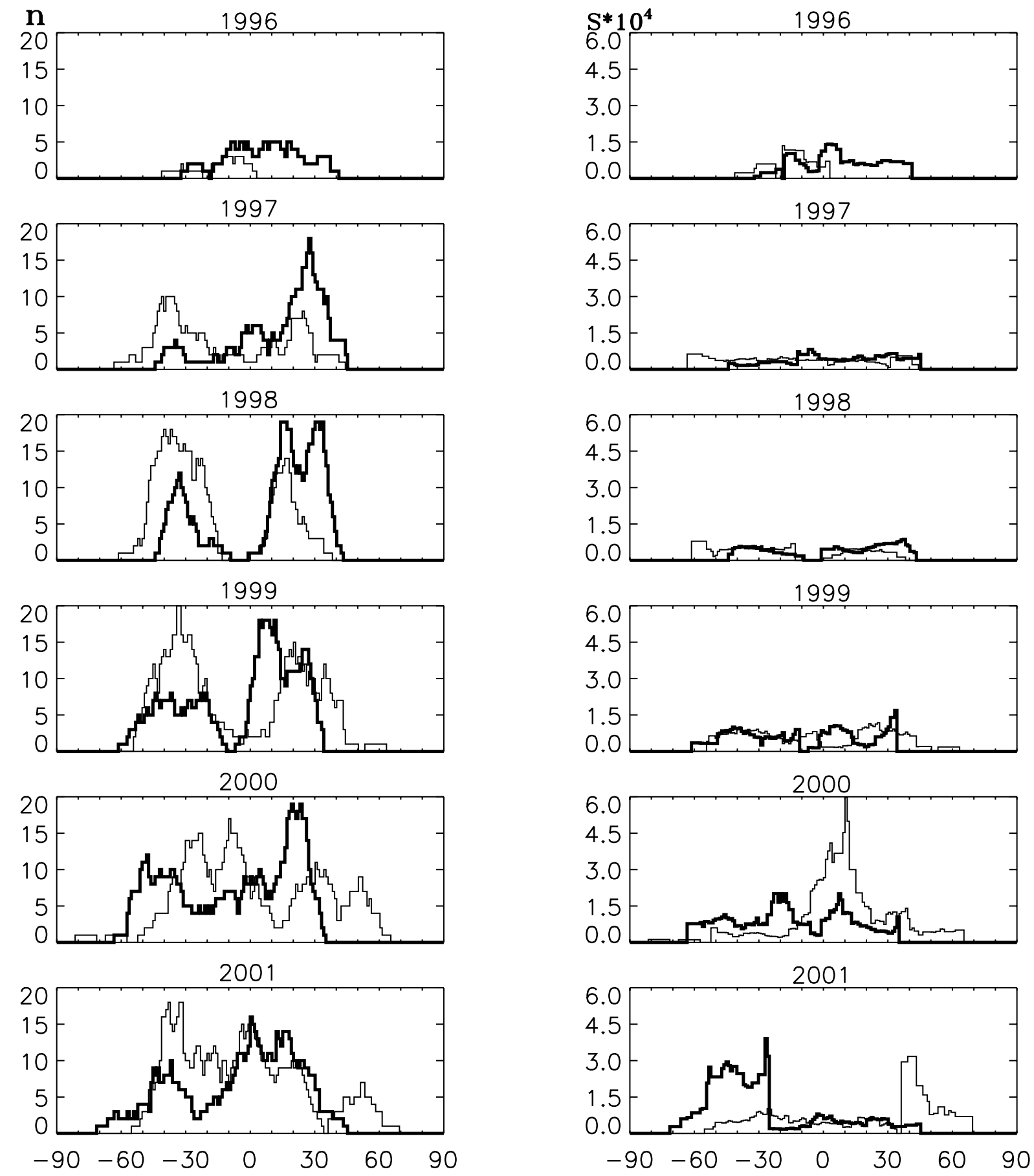

Fig. 4. The yearly average coronal hole number $n$ (left column) and the approximate areas $S$ (right column) for coronal holes corresponding to positive (thick line) and negative (thin line) photospheric magnetic fields for every year in one degree latitudinal intervals. Years are indicated on the top of each graphic.

longitudes of opposite polarity, covering the whole solar disk, has been seen since the end of 1998 (Fig. 2). The alternating mode means that one side of the Sun is covered by the non-polar coronal holes associated with the positive-polarity photospheric magnetic fields and the other is covered by the non-polar coronal holes associated with the negative-polarity photospheric magnetic fields. This mode reflects the sectorial photospheric magnetic field structure that existed since the end of 1998.
The transfer to this mode took a short period of time, about $1 \div 2$ solar rotations. Coronal holes of the same polarity are often connected by enhanced unipolar photospheric magnetic field regions in both hemispheres (Fig. 3, 02/24/01). The alternating regime reflects a sector magnetic field structure. Redistribution of positive-polarity and negative-polarity coronal holes took place inside these unipolar sectors (Figs. 2, 3). Coronal hole evolution indicates redistribution of positive and negative polarity photospheric magnetic fields inside these 
longitudinal sectors. Inside these sectors the redistribution of latitudinal locations and areas of positive coronal holes inside the positive sectors and of negative coronal holes inside the negative sectors took place. New positive coronal holes inside the positive sectors appeared at higher latitudes and long-lived coronal holes moved to the pole of opposite polarity.

The shape of coronal holes was deformed due to the differential rotation and in high latitudes the near-polar part of the negative-polarity sector coronal hole extended and encircled the north pole (Fig. 3 left column). They surrounded the pole and created a new polarity polar coronal hole. Even after the northern magnetic pole polarity change, the negativepolarity coronal holes continue to exist in these sectors both in the north and in the south hemispheres (Fig. 3).

The new north polar coronal hole has been observed since February 2001. The north polar region did not become completely encircled by a negative-polarity hole until about April 2001. The new north polar coronal hole is asymmetric with large projections. This new-polarity coronal hole covers the north pole over a wide range of longitudes. Since August 2001 the new polar coronal hole has covered all the solar north pole and became more symmetric. In the south hemisphere the polar reversal took place at the beginning of 2002, approximately 11 months after the north polar field reversal. The subsequent evolution of the coronal holes associated with the positivepolarity photospheric magnetic field in the positive sectors is shown in Fig. 3, right side. A long-term reversal to new-cycle polarity (negative in the north, positive in the south) occurred above latitudes $60^{\circ}$ around February 2001 in the north and about 11 months later in the south. The new polarity coronal hole has been existed on the south pole since May 2002.

One of the characteristics of the coronal hole evolution was the area increase and poleward growth of particular non-polar holes of new-cycle polarity, to eventually cover the poles. Areas of coronal holes associated with negative-polarity photospheric magnetic fields increased in the northern hemisphere and those of the positive-polarity in the south in the unipolar coronal hole sectors existing at that time. Areas were calculated for every coronal hole in millionths of the solar hemisphere. Figure 4 shows the yearly average coronal hole numbers $n$ (left column) and their approximate areas $S$ (right column) for coronal holes corresponding to positive (thick line) and negative (thin line) photospheric magnetic fields for every year in one degree latitude intervals. Figure 4 shows that the coronal hole number and area distributions follow the solar polar field reversal.

In 1996, at the solar cycle minimum, coronal holes concentrated in the equator region and their areas were of maximum size at the equator. Coronal holes associated with the positive photospheric magnetic fields were located and their area were maximum at $-30^{\circ} \div 40^{\circ}$, and coronal holes associated with negative photospheric magnetic fields were located and their areas were of maximum size in the region of $-40^{\circ} \div 3^{\circ}$ of latitudes. In 1997-1998 the number and areas of positive-polarity coronal holes were maximal in the north hemisphere and the number and areas of negative-polarity coronal holes were maximal in the south hemisphere. In 1999 the negative-polarity coronal holes were located at $-55^{\circ} \div 65^{\circ}$ of latitude and positivepolarity at $-60^{\circ} \div 35^{\circ}$. The maximum of the positive-polarity

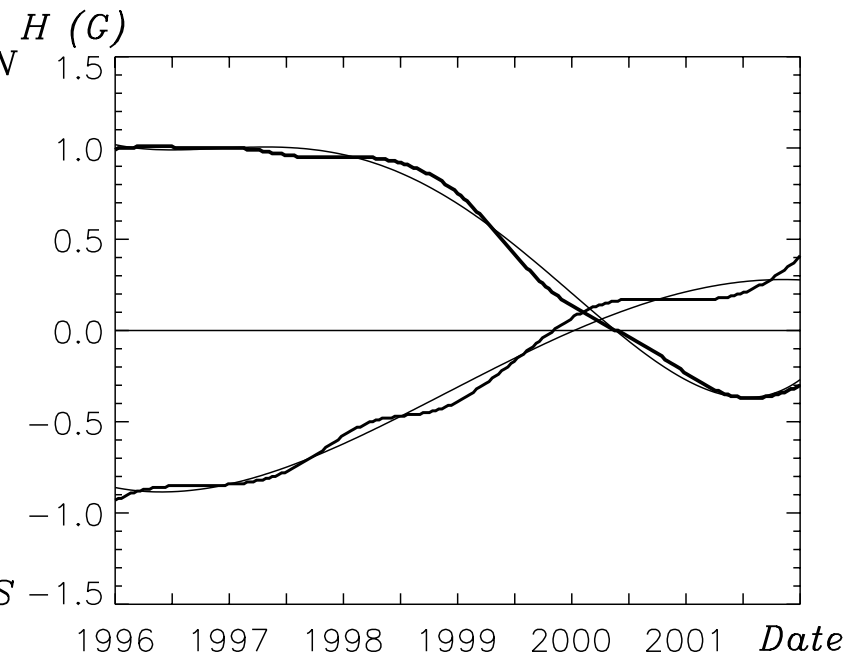

Fig. 5. The variation of the northern $(\mathrm{N})$ and southern $(\mathrm{S})$ photospheric magnetic polar field strength for 1996-2001 (heavy lines). These data are from the Wilcox Solar Observatory. The thin solid line is a fourth degree polynomial fit.

coronal hole area was at $35^{\circ}$ in the south hemisphere and about $15^{\circ}$ in the north hemisphere. The maximum of the negativepolarity coronal hole area was at $-30^{\circ}$ in the south hemisphere and about $25^{\circ}$ in the north hemisphere. In 2000 positivepolarity coronal holes were located at $-65^{\circ} \div 35^{\circ}$ and their maximum areas were in the south hemisphere and the negativepolarity coronal holes were located at $-55^{\circ} \div 70^{\circ}$. Their maximum areas were in the north hemisphere at about $15^{\circ}$. In 2001 the positive-polarity coronal holes were located at $-70^{\circ} \div 45^{\circ}$, their maximum areas were at $-70^{\circ} \div-30^{\circ}$; negative-polarity coronal holes were located at $-55^{\circ} \div 70^{\circ}$, the maximum areas were at $35^{\circ} \div 70^{\circ}$.

Although the polar magnetic fields are weak compared to the fields of active regions, their strength variation suggests that they are an important component of the solar activity cycle. The polar field is nearly radial. The polar fields are strongest and polar coronal holes are of maximum area at solar minimum. Near sunspot maximum the polar field weakens and reverses polarity (Babcock 1959; Svalgaard 1978). The time of the maximum polar coronal hole area is half a cycle in antiphase with the peak of the sunspot number (Bravo \& Steward 1997; Sanchez-Ibarra 1990). Figure 5 shows the variation of the polar photospheric magnetic field strength for both the north pole and the south pole for the time period 1996-2001 (Wilcox Solar Observatory). The first period of the coronal hole distribution coincides with the maximum value of the polar field strength. The magnetic field strength reaches its minimum value in the south polar cap earlier than in the north one (Fig. 5). The sectorial structure appears when the polar field strength reaches 0.7 , its maximum value in the corresponding hemisphere.

Thus the coronal hole evolution reflects the evolution of photospheric magnetic fields. It is interesting to analyze the change in the magnetic fields. As was mentioned before, coronal holes are associated with unipolar magnetic regions. In order to determine the geometric structure of the global solar magnetic field, a spherical harmonic analysis technique was 
developed by Altschuler \& Newkirk (1969) and Altschuler et al. (1975). This technique finds the harmonic coefficients that give the best least-mean-square fit to the measured line-ofsight component of the photospheric magnetic field as a boundary condition. The assumption of a current-free magnetic field configuration is used. The smaller details are represented by the harmonics of higher order. The harmonic coefficients for 19041984 Carrington rotations (1996-2001 years) from the Wilcox Solar Observatory were used.

The solar magnetic field may be described as a function of latitude and longitude coordinates $(r, \theta, \phi)$ (Altschuler \& Newkirk 1969; Chapman \& Bartels 1940)

$$
\begin{aligned}
\psi(r, \theta, \phi)= & R \sum_{n=1}^{N} \sum_{m=0}^{n}\left(\frac{R}{r}\right)^{n+1}\left(g_{n}^{m} \cos m \phi\right. \\
& \left.+h_{n}^{m} \sin m \phi\right) P_{n}^{m}(\theta),
\end{aligned}
$$

where $P_{n}^{m}(\theta)$ are the associated Legendre Polynomials, $N$ is the largest number of harmonics. Index $n$ determines the multipole moment of the harmonic; $n=0$ is a monopole, $n=1$ is a dipole, $n=2$ is a quadrupole, $n=3$ is an octopole, and $n=N$ is a "2 $2^{N}$-pole". The coefficients $g_{n}^{m}$ and $h_{n}^{m}$ must be determined from the measurements of the line-of-sight component of the magnetic field at the boundary surface $r=R$. The magnetic field components are the gradients of the solar potential $\psi$. The formula represents the solar magnetic field as the sum of simple functions. Each harmonic, identified by its indices $(n, m)$, represents the contribution of a particular geometric structure to the overall solar field distribution. The smaller details of the magnetic field are described by the higher harmonics, with greater numbers of $n$ and $m$.

The pure latitudinal structure of the solar magnetic fields is described by harmonics with index $m=0$. These functions are called zonal spherical harmonics. Functions become zero in $m$ parallels and divide the solar surface into zones where they have positive and negative values. They describe the rotationaxis symmetrical components of the solar magnetic field. When $n$ is odd, the symmetry is about the equatorial zone. If $n$ is even then the symmetry is relative to the equator plane. There are $n-m+1$ zones.

When $n=m$ the functions are called sectorial; that is, there are no zeros along a meridian of longitude. The regions of constant sign are sectors of the sphere. They have alternately positive and negative values in the sector structures limited by meridians. When $n-m \neq 0$, the harmonics are called tesseral. They have alternately positive and negative values in spherical quadrangles or in spherical triangles in the polar regions. We assume that there is no monopole component for the solar magnetic field.

The multipole power spectrum gives the relative energy contribution of each multipole moment of the field. The largest value of $S_{n}^{m}$ determines the single dominant harmonic in a set of coefficients. Various power spectra can be calculated (Altschuler et al. 1977; Levine 1977). The harmonic spectrum is defined as

$S_{n}=\sum_{m=0}^{n}\left[\left(g_{n}^{m}\right)^{2}+\left(h_{n}^{m}\right)^{2}\right]$.
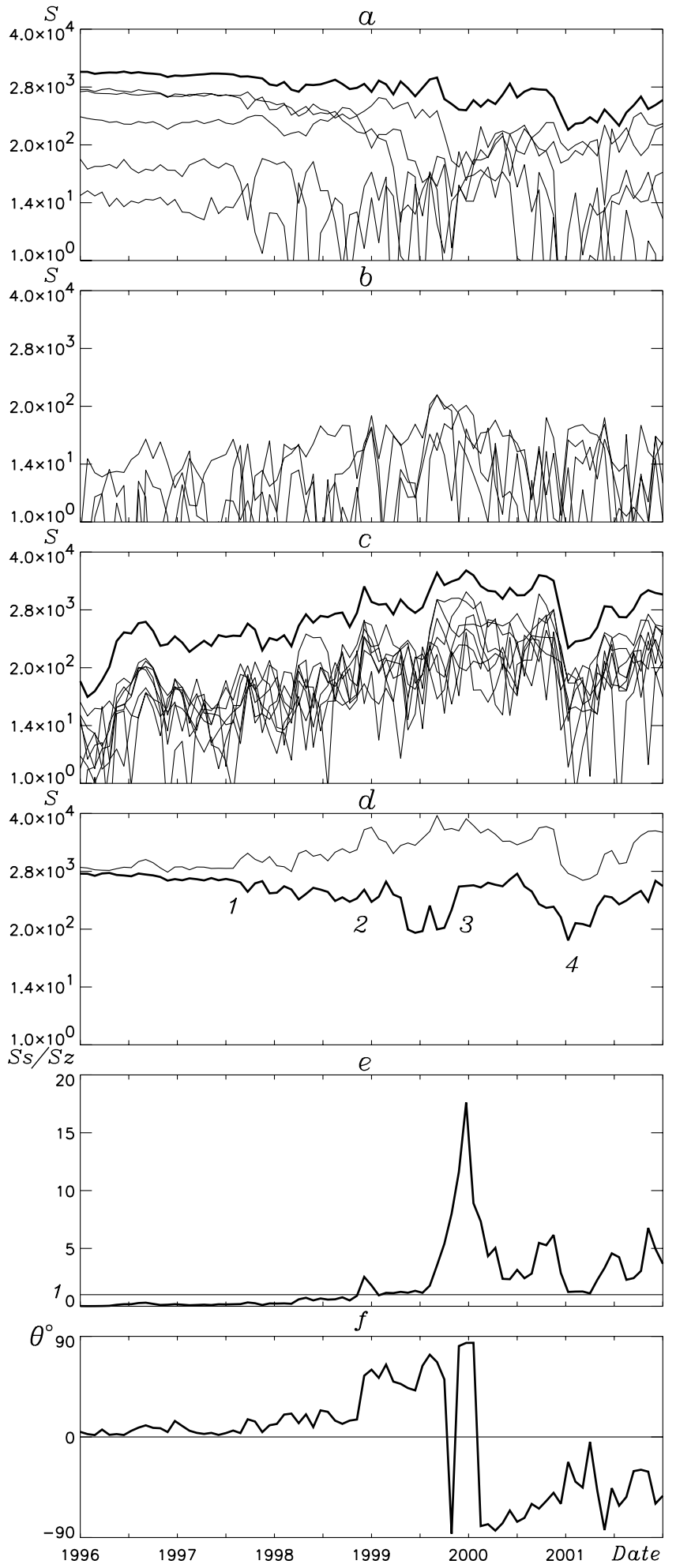

Fig. 6. Multipole power spectra of the solar photospheric magnetic field for CR 1904-1982 (1996-2001 years). a) The heavy line is the sum of zonal harmonic spectra. The thin lines are the zonal harmonic spectra for odd $n(n=1,3, \ldots, 9)$. b) The zonal harmonic spectra for even $n(n=2,4, \ldots, 8)$. c) The heavy line is the sum of the sectorial harmonic spectra. The thin lines are the sectorial harmonic spectra for different $n=m$. d) The heavy line is the dipole component $(n=1)$ and the thin line is the sum of multipoles of the harmonic spectra. e) Ratio of the sectorial harmonic spectra sum to the zonal harmonic spectra sum. f) The polar angle of the dipole component. 
The polar angle, $\theta$, of the dipole component of the photospheric magnetic field is defined as

$\tan \theta=\left(g_{1}^{0}\right)^{-1}\left[\left(g_{1}^{1}\right)^{2}+\left(h_{1}^{1}\right)^{2}\right]^{1 / 2}$.

Figure 6 shows the multipole power spectra $S_{n}^{m}$ of the solar photospheric magnetic field for CR 1904-1982 (1996-2001). The sum of zonal harmonic spectra $(m=0)$ is shown in Fig. 6a (heavy line). The zonal harmonic spectra for odd $n$, $n=1,3, \ldots 9$, are shown by thin lines in Fig. 6 a and those for even $n(n=2,4, \ldots, 8)$ are shown in Fig. 6b. The sum of the sector harmonic spectrum $(n=m)$ is shown in Fig. 6c (heavy line). Thin lines show sector harmonic spectra for different $n=m(n=1,2, \ldots, 9)$. Figure $6 \mathrm{~d}$ shows the dipole component ( $n=1)$ of the harmonic spectrum (heavy line) and the harmonic spectrum for the sum of multipole components $(n=2,3, \ldots, 9)$ (thin line). The ratio of the sum of sectorial harmonics to the sum of zonal harmonics is shown in Fig. 6e. The polar angle of a dipole component of the photospheric magnetic field is shown in Fig. 6f. The Sun's magnetic field structure is complex and dynamic. Each of these power spectra represent the decomposition of the same total field into different types of geometric patterns. There are periods when the geometrical structure changes significantly. These can be interpreted as periods of relatively sudden rearrangements of the multipole structure. The first period of the coronal hole distribution is dominated by the zonal geometry (Figs. 1, 6a, 6e). The dipole component of the magnetic field has its maximum value (Fig. 6d). The polar field strength is maximum (Fig. 5). The polar angle is equal to the minimum value. Point 1 (Fig. 6d) indicates the beginning of the second period of coronal hole distribution on the solar disk. This moment coincides with the moment when the sum of multipole components of magnetic fields begins to increase and the dipole component begins to decrease. The sectorial structure is established on the whole solar disk from the end of 1998 (point 2 in Fig. 6d). The sum of the sectorial harmonics dominate from that time on (Fig. 6e). The third period of the coronal hole distribution began at the end of 1998 (point 2 in Fig. 6d). The sectorial harmonics began to dominate (Fig. 6e). Point 3 (Fig. 6d) corresponds to the moment when the northern and southern photospheric magnetic polar field strengthes are equal. At that time the dipole component reached its minimum value, the sum multipole component was maximum (Fig. 6d), zonal harmonic spectra for $n=1,3,5$ had their minimum values (Fig. 6a), zonal harmonic spectra for even $n$ (Fig. 6b) and sector harmonic spectra (Fig. 6c) reached their maxima. The polar angle of the dipole component of the photospheric magnetic field increased to $\approx 90^{\circ}$ (Fig. 6e). At the beginning of 2000 it changed the sign and began to decrease. These rearrangements in the multipole geometry of the magnetic field coincide with the changes of the solar polar magnetic field strength and the coronal hole distribution transfer from the zonal structure to the sectorial structure. The moment of the new-polarity north polar coronal hole creation coincides with point 4 , Fig. $6 \mathrm{~d}$. The polar angle had its minimum value (Fig. 6f) and the input of zonal and sectorial components were equal (Figs. 6c,e). The coronal hole evolution described above, and its relationship to the photospheric magnetic field evolution and reversal of the polar magnetic fields suggest that they may have a more direct connection with solar cyclic processes. It would appear that the large-scale magnetic fields in the polar regions, which dominate the poloidal component of the large-scale solar magnetic field, are not solely the product of decaying active regions.

\section{Conclusions}

1. It seems that the coronal holes were not distributed randomly during 1996-2001. There are three different periods of non-polar coronal hole distribution on the solar disk. During the solar minimum they are located in the equator region and coronal holes associated with the positivepolarity photospheric magnetic field drift toward the south pole and coronal holes associated with the negative-polarity photospheric magnetic fields drift toward the north pole. The first period of coronal hole distribution coincides with the maximum value of the polar field strength. The dipole component of the global solar magnetic field dominated at that time. During the second period the polarity of the coronal-hole-associated photospheric magnetic fields matched the polarity of the polar field in that hemisphere in general. During the third period, longitudes covered by non-polar coronal holes of the opposite polarity alternated. The alternating regime reflects a sectorial global magnetic field structure. This structure was established in both hemispheres at different times. In the north hemisphere it has been present since November 1998 and in the south hemisphere since October 1997. The sectorial structure appears when the polar field strength reaches 0.7 its maximum value in the corresponding hemisphere. The rearrangements from one configuration to another occur over a short time period of about $1 \div 2$ solar rotations. The magnetic field strength reaches its minimum value in the south polar cap earlier than in the north one.

2. Polar and non-polar coronal holes are connected in their evolution. Projections from the polar coronal holes are often directed to the non-polar coronal holes associated with the photospheric magnetic fields of the same polarity at low latitudes. Sometimes parts of polar coronal holes separate and form independent coronal holes or non-polar coronal holes unite with the polar coronal hole. The dynamics of polar and non-polar coronal holes lead to the conclusion that they have a common nature.

3. The process of polarity reversal accompanying the redevelopment of the polar holes is not a smooth process that sweeps the trailing flux from the emerging flux belts to the pole. Instead it is discontinuous, occurring in unipolar longitude sectors. Coronal hole number and area evolution indicates redistribution of positive-polarity and negativepolarity photospheric magnetic fields inside these longitudinal sectors. This reflects the process of change of dominant polarity. Near sunspot maximum the polar field weakens and reverses polarity. In cycle 23 the polar polarity reversal and polar hole redevelopment occurred first in the north. There is an asymmetry in the polar polarity reversal between the two hemispheres; the polarity reversal in 
the two hemispheres is offset by approximately 11 months. In the northern hemisphere the new negative-polarity polar coronal hole was created at the beginning of 2001 and in the southern hemisphere the new positive-polarity polar coronal hole was created at the beginning of 2002.

4. Evolution of the solar magnetic field goes from a dominant dipole field, almost parallel to the solar rotation axis, to a highly inclined dipole and higher-order multipole. Coronal holes are a manifestation of this structure evolution. The phases of coronal holes and solar magnetic field evolution and solar polar field reversal that are described in the paper indicate that the evolution of the coronal holes is part of a general evolutionary process that is related to the Sun's global magnetic field.

Acknowledgements. I am grateful to Dr. I. S. Veselovsky for helpful discussions. NSO/Kitt Peak data used here were produced cooperatively by NSF/NOAO, NASA/GSFC, and NOAA/SELL. I would like to thank Wilcox Solar Observatory, and Dr. J. Todd Hoeksema for making available the harmonic coefficient data used in this study.

\section{References}

Altschuler, M. D., \& Newkirk, G. Jr. 1969, Sol. Phys., 9, 131

Altschuler, M. D., Trotter, D. E., Newkirk, G. Jr., \& Howard, R. 1975, Sol. Phys., 41, 225

Altschuler, M. D., Levine, R. H., Stix, M., \& Harvey, J. 1977, Sol. Phys., 51, 345

Babcock, H. D. 1959, ApJ, 130, 364

Belenko, I. A. 2001, Sol. Phys., 199, 23
Bohlin, J. D., \& Sheeley, Jr. N. R. 1997, Sol. Phys., 173, 193

Bravo, S., \& Steward, G. A. 1997, Sol. Phys., 173, 193

Bumba, V., Klvan̆a, M., \& Sýkora, J. 1995, A\&A, 298, 923

Chapman, S., \& Bartels, J. 1940, Geomagnetism, V.2, Oxford

DeVore, C. R., \& Sheeley, N. R. Jr. 1987, Sol. Phys., 108, 47

Fox, P., McIntosh, P., \& Wilson, P. R. 1998, Sol. Phys., 177, 375

Harvey, K. L., Sheeley, N. R., Jr., \& Harvey, J. W. 1982, Sol. Phys., 79,149

Ikhsanov, R. N., \& Ivanov, V. G. 1999, Sol. Phys., 188, 245

Kulěár, L., \& Sýkora, J. 1994, Contrib. Astron. Obs. Skalnate Pleso, 24,79

Leighton, R. B. 1964, ApJ, 140, 1547

Levine, R. H. 1977, Sol. Phys., 54, 327

Levine, R. H. 1982, Sol. Phys., 79, 203

Makarov, V. I., Fatianov, M. P., \& Sivaraman, K. R. 1983, Sol. Phys., 85,215

Makarov, V. I., \& Sivaraman, K. R. 1983, Sol. Phys., 85, 227

Makarov, V. I., \& Sivaraman, K. R. 1989, Sol. Phys., 119, 35

Makarov, V. I., \& Tlatov, A. G. 2000, Astron. Rep., 44, 11, 759

Obridko, V. N., \& Shelting, B. D. 1999, Sol. Phys., 187, 185

Sanchez-Ibarra, A. 1990, Sol. Phys., 125, 125

Sanchez-Ibarra, A., \& Barraza-Paredes, M. 1992, Report UAG-102 WDCA, Boulder, USA

Sheeley, N. R. Jr., Nash, A. G., \& Wang, Y. M. 1987, ApJ, 319, 481

Svalgaard, L., Duvall, T. L. Jr., \& Scherrer, P. H. 1978, Sol. Phys., 58, 225

Timothy, A. F., Krieger, A. S., \& Vaiana, G. S. 1975, Sol. Phys., 42, 135

Varsik, J. R., Wilson, P. R., \& Li, Y. 1999, Sol. Phys., 184, 223

Waldmeier, M. 1981, Sol. Phys., 70, 251

Webb, D. F., Davis, J. M., \& McIntosh, P. S. 1984, Sol. Phys., 92, 109 